\title{
Preferencias de la demanda sobre los materiales de construcción del inmueble residencial: caso de estudio con el Análisis Conjunto Adaptativo
}

Silvia Spairani Berrio ${ }^{1} \mid$ Josep Roca Cladera ${ }^{2}$

Recibido: 10-03-2020 | en su versión final: 14-05-2020

Resumen

\begin{abstract}
En este artículo se pretende, presentar y describir la metodología de una técnica aplicativa híbrida del Análisis Conjunto, denominada Análisis Conjunto Adaptativo (ACA), desarrollada por Johnson (1987) para mejorar la complejidad del experimento al limitar el número de perfiles que se presenta al entrevistado y distribuida por el programa Sawtooth (1991). A tales efectos dicho método ha sido empleado para realizar, en la provincia de Alicante, una evaluación científica de la valoración estandarizada, de la estimación de las tasas marginales de sustitución de la utilidad de los materiales de construcción de los inmuebles residenciales, para cuantificar el cambio de percepción del participante provocado por un cambio de elección de material. Desde el punto de vista operativo, la estimación de la importancia relativa de los atributos de los materiales de construcción evaluados, utilizando el ACA, muestra el gran impacto que la localización del material en la vivienda tiene sobre el comportamiento de elección de los consumidores. En el recorrido efectuado por la bibliografía no se ha encontrado ninguno que reflexione, en España, en la utilización de esta técnica para la realización de estudios que permitan mejorar tanto el cálculo de la estimación del valor de mercado de un inmueble residencial como su uso en arquitectura a través de los materiales de construcción. Este trabajo, por tanto, es pionero en estos sentidos.
\end{abstract}

Palabras clave: ACA; preferencias del consumidor; comportamiento del consumidor; percepción de la calidad de los materiales de construcción

Citación

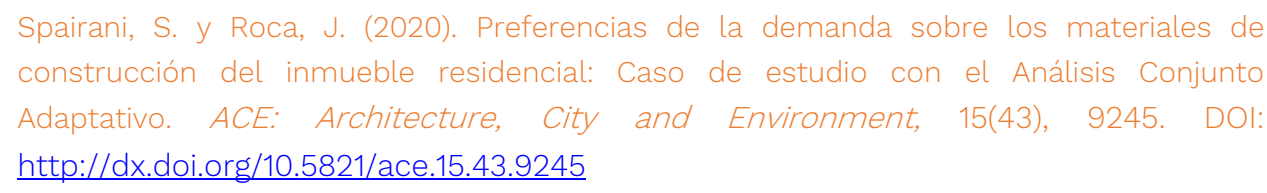

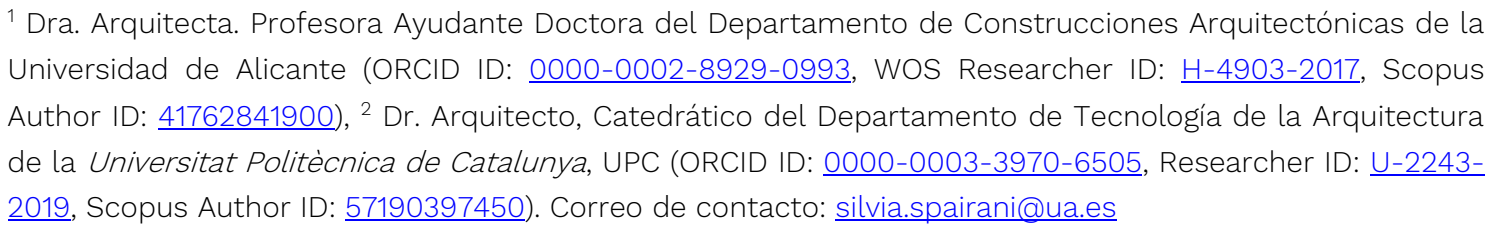

ACE, 15 (43) CC BY-ND 3.0 ES | UPC Barcelona, España | Preferencias de la demanda sobre los materiales de construcción del inmueble residencial: caso de estudio con el Análisis Conjunto Adaptativo. DOI: http://dx.doi.org/10.5821/ace.15.43.9245 


\section{Demand preferences on residential building materials: Case study with Adaptive Conjoint Analysis}

Abstract

This article aims to present and describe the methodology of a hybrid application technique of the Conjoint Analysis, called Adaptive Conjoint Analysis, developed by Johnson in 1987 to improve the complexity of the experiment by limiting the number of profiles presented to the interviewee and distributed by Sawtooth Software in 1991. To this end, this method has been used to carry out, in the province of Alicante, a scientific assessment of the standardized assessment, of the estimation of marginal rates for replacing the usefulness of residential building materials, to quantify the participant's change in perception caused by a change in material choice. From an operational point of view, estimating the relative importance of the attributes of the building materials evaluated, using the ACA, shows the great impact that the location of the material on the home has on the behavior of consumer choice. In the journey through the literature, none have been found that reflect, in Spain, in the use of this technique to carry out studies that allow improvement both the calculation of the estimation of the market value of a residential property and its use in architecture through building materials. This work, therefore, is a pioneer in these ways.

Keywords: ACA; consumer preferences; consumer behavior; perception of the quality of building materials

\section{Introducción}

Recientemente en España la herramienta de software del modelo multi-atributo del análisis conjunto ${ }^{1}$ permite conocer con profundidad y rigor técnico las preferencias de percepción de utilidad de los consumidores para respaldar tanto medidas de toma de decisiones urbanísticas y de diseño inmobiliario (Marmolejo y Ruiz, 2013; Marmolejo y Ruiz, 2014; Marmolejo, Olivares, Montero y De Paolini, 2014) como de eficiencia energética. Respecto a este último destaca el Proyecto EnerValor (MINECO/FEDER-2015-63606-R) liderado por el Dr. Arq. Carlos Marmolejo Duarte que utiliza para una parte del desarrollo de su investigación el análisis conjunto basado en elección (CBC) (Marmolejo y Bravi, 2017; Spairani et al, 2019; Marmolejo, García y Spairani, 2020). Todo ello, con el objetivo de conocer la estructura de preferencias de los hogares en referencia a la importancia relativa que le dan a la eficiencia energética en la elección residencial, convirtiéndose en una ventaja competitiva y que permite ir hacia un modelo urbanístico y arquitectónico de desarrollo sostenible.

Asimismo, en España, respecto a las preferencias de la demanda sobre los materiales de construcción del inmueble residencial son pioneros los estudios de (Spairani y Roca, 2013 y Spairani,

\footnotetext{
${ }^{1}$ El Conjoint Analysis o Análisis Conjunto (AC) define un conjunto de metodologías estadísticas especialmente dirigidas al estudio de los modelos de elección del consumidor a partir de juicios de preferencia expresados por estos últimos en relación con diferentes perfiles de un producto y/o servicio a desarrollar (Gustafsonn et al., 2001; Hensher et al., 2005). Teóricamente el AC es un modelo de análisis de varianza de los principales efectos de los atributos sobre la utilidad, siendo equivalente a un análisis de regresión de un índice de preferencia sobre los niveles medidos de un atributo.
} 
2016), dado que han realizado en la Comunidad Valenciana una investigación que profundiza en el cálculo de un índice sintético compuesto de calidad percibida de los materiales de construcción para el uso residencial, centrándose en la problemática de la percepción subjetiva del consumidor a través del modelo multi-atributo del Análisis Conjunto Adaptativo (ACA).

Tradicionalmente, la medida de la calidad percibida se ha centrado en buscar, en sentido general, el equilibrio respecto a la utilidad de los distintos atributos de la percepción del participante que cumplan una función de satisfacción de sus preferencias (Thaler, 1985). No cabe duda que, al mezclarse percepciones conscientes e inconscientes ${ }^{2}$, la percepción del individuo no es real, no siendo posible utilizar un modelo compensatorio de evaluación (Som y Lee, 2012; Huertas, Gázquez, Martínez y Esteban, 2014), entre otras cuestiones porque sus emociones afectan a las percepciones de satisfacción ${ }^{3}$. Esta visión sobre la percepción de la satisfacción es la utilizada por el participante en el momento en que toma la decisión de compra (Grönroos, 1984), pudiendo obviar alternativas que le reporten una mayor utilidad. De ahí que, resulte extremadamente difícil de cuantificar el comportamiento del consumidor, a causa de las diferencias en la conducta del participante durante el proceso de búsqueda de información, el cual debe permitirle evaluar las alternativas (o atributos) ${ }^{4}$ que ofrece el mercado y elegir la que más satisfaga sus deseos (Kahneman y Tversky, 1979; Lancaster, 1990). Este enfoque permite estimar los costos y beneficios asociados con los atributos de ubicación que la demanda considera importante cuando adquiere una vivienda. Sin embargo, su dificultad radica en identificar un patrón de comportamiento que permita modelizar adecuadamente la toma de decisión del participante.

En este contexto, las preferencias de la demanda sobre los materiales de construcción empleados en las viviendas son vistos como un conjunto de atributos que, cuando son estratégicamente ubicados en el inmueble, permiten ajustarse a las necesidades y preferencias de la demanda. No cabe duda que el sistema de preferencia del consumidor es de hecho, una preferencia de medición de naturaleza compleja, dado que los consumidores presentan distintas características que les diferencian, siendo la percepción de la calidad ${ }^{5}$ de un del producto la representación de una subconducta durante el proceso de decisión ${ }^{6}$ de compra (Lambin y Peeters, 1981). De acuerdo con esta teoría, la utilidad del material de construcción de la vivienda puede descomponerse en un componente sistemático observable y un componente aleatorio no observable, que incluye al término de error.

Consecuentemente, el modelo multi-atributo debe considerar y permitir incluir tanto el atractivo estético de la calidad de los materiales de construcción (CES), como la calidad técnica de dichos

\footnotetext{
2 Estas percepciones posiblemente provienen de las imágenes que hacen referencia a otras experiencias, no siempre consecuentes. Por ello, no hay que minusvalorar la aportación inconsciente en la calidad del material, ni se debe eludir todo aquello que no se pueda explicar racionalmente.

${ }^{3}$ La felicidad, el placer, el júbilo, son ejemplos que proporcionan una satisfacción positiva al consumidor, mejorando siempre su percepción. En sentido contrario, actuaría la insatisfacción que le podría dar la tristeza, pena, irritación, etc.

4 Se entiende desde el punto de vista psicológico ya que es una propiedad extraída de la experiencia humana, atribuyéndose a alguna cosa y no a la cosa misma (relacionándose con la percepción que tenemos de una característica o propiedad del producto).

${ }^{5}$ Valoración global que realiza el consumidor de un material de construcción y que le reportará una satisfacción de sus expectativas, en base a ciertos atributos condicionantes en su decisión de elección de un inmueble residencial, que diferirá de la evaluación realizada por otro consumidor considerando, el momento situacional, la ubicación del material, la renta de la que disponga y el perfil socio-demográfico que le caracteriza.

${ }^{6}$ El cual está formado por una secuencia de actividades de procesamiento de información (procesos cognitivos recibidos del mundo que rodea al consumidor a través de los 5 sentidos, siendo dicho proceso dependiente tanto de la índole del estímulo físico como de la relación de éste con el ambiente que lo rodea) y, por una secuencia de comportamientos derivados de la adquisición y la evaluación de la información que van conformando, a medida que el proceso de decisión de compra se desarrolla (condiciones internas del consumidor).
} 
materiales $(\mathrm{CTO})^{7}$ En este sentido, de entre todas las alternativas de consumo posible, la demanda elige aquella que le proporciona el máximo nivel de utilidad (o satisfacción) de acuerdo con sus expectativas (Johnson y Meyer, 1984; Hogarth, 1987, Zeithaml, 1988; Goodin, 1995; Simonotti, 2006). Paralelamente, el precio debe considerarse como un atributo capaz de presentar un elevado grado de correlación con las características de la alternativa (Robinson, 1980; Johnson y Olberts, 1991), analizándose así en el ejemplo del aplicativo mostrado en este artículo. En otras palabras, se ha tenido en cuenta que a pesar de ser el precio ${ }^{8}$ un atributo extrínseco, tiene suficiente entidad por sí mismo para desligarse del resto. Entre otras cuestiones porque el precio puede presentar características implícitas representativas de la calidad del producto (Oliphant, Eagle, Louviere y Anderson, 1992) y que ocasionalmente suman o restan utilidad final al atributo. Un aumento en el precio final del producto será visto en ocasiones favorablemente y en otras desfavorables, conllevando a que la percepción de utilidad del mismo satisfaga sobradamente todas sus necesidades y expectativas, o que en caso contrario provoque descontento sobre éste.

Los principales estudios publicados en revistas indexadas en los diferentes índices de ISI y Scopus en el último decenio, en relación al análisis conjunto y sus aplicaciones en el ámbito de la arquitectura y urbanismo en otros países se centran en el análisis conjunto: tradicional (Bae, 2006; Macias, y Knowles, 2011; Zhang y Zhu, 2012; Lee et al., 2014; Alaraji y Jusan, 2014; Fitri et al., 2017; Franke y Nadler, 2019), con experimentos de elección (Morrow, Irwin y Roe, 2004; Timmermans, y Katoshevski, 2011; Jay y Bowen, 2011; Nordh, 2012; Arming y Ziefle, 2015; Mansour y Radford, 2016), con logit multinomial (Rofe et al., 2017; Maeng et al., 2019) y jerárquico (Banfi et al., 2008; Goossen, y Langers, 2000; Hagishima et al., 2012). Se resumen en la siguiente Tabla 1 los correspondientes al análisis conjunto adaptativo. Como se ve, todos ellos corresponden a un análisis de la arquitectura asociada a viviendas.

Tabla 1. Selección de estudios que han aplicado el ACA en el ámbito de la arquitectura-urbanismo

\begin{tabular}{|c|c|c|c|c|c|}
\hline Autor & Año & Ámbito temático & País & $\begin{array}{l}\text { Tipo de } \\
\text { análisis } \\
\text { conjunto }\end{array}$ & Finalidad \\
\hline Spairani, S & 2016 & $\begin{array}{l}\text { Análisis de la calidad } \\
\text { percibida de los } \\
\text { materiales de } \\
\text { construcción para el } \\
\text { uso residencial }\end{array}$ & España & $\mathrm{ACA}$ & $\begin{array}{l}\text { Cálculo de un índice sintético } \\
\text { compuesto de calidad percibida } \\
\text { de los materiales de construcción } \\
\text { para el uso residencial, } \\
\text { centrándose en la problemática } \\
\text { de la percepción subjetiva del } \\
\text { consumidor }\end{array}$ \\
\hline $\begin{array}{l}\text { Hildebrandt, J. } \\
\text { Brauner, P. y } \\
\text { Ziefle, M. }\end{array}$ & 2015 & $\begin{array}{l}\text { Análisis de las } \\
\text { preferencias } \\
\text { electrónicas con } \\
\text { fines de mejora de } \\
\text { los hogares } \\
\text { inteligentes }\end{array}$ & Alemania & $\mathrm{ACA}$ & $\begin{array}{l}\text { Análisis de la integración de la } \\
\text { electrónica en la arquitectura } \\
\text { textil }\end{array}$ \\
\hline $\begin{array}{l}\text { Heinzle, S.L. } \\
\text { Boey, A. y Low, } \\
\text { M. }\end{array}$ & 2013 & $\begin{array}{l}\text { Valoración marca } \\
\text { verde en inversiones } \\
\text { inmobiliarias } \\
\text { residenciales }\end{array}$ & Singapur & $\mathrm{ACA}$ & $\begin{array}{l}\text { Análisis de la influencia de los } \\
\text { esquemas de certificación de } \\
\text { edificios eficientes en el } \\
\text { comportamiento de los } \\
\text { inversores inmobiliarios respecto } \\
\text { a la arquitectura residencial }\end{array}$ \\
\hline
\end{tabular}

Fuente: Elaboración propia

\footnotetext{
7 Se parte de la hipótesis que la calidad percibida de los materiales (CPM) no es una función de la calidad técnica del material, sino una interrelación compleja de las variables (СTO), que dependerá de la estancia del inmueble donde se ubique, y de la variable (CES). CPM=f[(CTO), (CES)]

${ }^{8}$ En la actualidad se da la posibilidad de utilizar la técnica del CBC del Sowtooth Software Inc, (1985-2010) para trabajar con análisis centrados en el precio, que tiene en cuenta todas estas interacciones.
} 


\section{Adaptive Conjoint Analysis}

Con el objetivo de hacer el AC más competitivo, el desarrollo de investigación sobre el perfeccionamiento de dicha técnica fue todavía más notable a partir de 1985. De ahí que no es de extrañar que en la búsqueda de la reducción del tamaño del diseño factorial, (Johnson, 1987) consiguió una técnica aplicativa híbrida de ésta mejorada, a la que denominó Adaptive Conjoint Analysis $(\mathrm{ACA})^{9}$. Siendo una combinación de un Self Explication Preference y de un método de confrontación de parejas (Paired Comparison Scale), es decir, combina en un solo cuestionario de evaluación preguntas para determinar la importancia de los atributos y de la comparación en parejas de perfiles formadas por los atributos valorados positivamente por los encuestados en la primera fase de la entrevista.

El término "adaptativo" se refiere al hecho de que la Software que realiza la entrevista se personaliza se va adaptando de forma secuencia al diseño experimental para cada entrevistado, en función de las preferencias declaradas en las comparaciones anteriores. Por ello, el encuestado responde en nuestra investigación según sus preferencias de elección entre las distintas posibilidades confrontadas de los atributos de los materiales de construcción, y para cada iteración parte de la explicación de los niveles de cada atributo evaluados en el confronte anterior para determinar la siguiente, hasta que el cuestionario de evaluación de la entrevista establece la utilidad global percibida por el encuestado sobre los atributos de la vivienda. Esta técnica basada en perfiles parciales se diferencia básicamente del método tradicional del CA, en la presentación de los atributos a los encuestados, ya que durante la entrevista tiene en cuenta las preferencias de elección previas. De esta forma, una de las principales fortalezas de esta metodología frente al análisis conjunto es que, durante su desarrollo, el análisis conjunto adaptativo explica más niveles de investigación al permitir mostrar al participante más comparaciones por pares, calculando la importancia relativa de cada atributo y obteniendo el nivel de utilidad real para la demanda (Johnson, 1989; Agarwal y Green, 1991). Por ello, no se trata simplemente de acortar la duración de la entrevista para evitar el posible cansancio del encuestado, sino de que el cuestionario se adapte al encuestado para las comparaciones sucesivas.

Esta metodología sirve pues, para paliar las excesivas contestaciones facilitadas por el encuestado cuando se daba un gran número de factores en la investigación a tratar. Según lo establecido por (Johnson, 1987, 1989 y 1991) el resultado será una concepción hibrida que combina los modelos conjuntos de componentes parciales de la utilidad total con los modelos auto-explicados, creando un conjunto aleatorio y reducido de estímulos (entre 3 y 9) seleccionados a partir de un diseño factorial fraccional, para evaluar un mayor número de atributos y de niveles que no sería factible en el AC de perfiles completos tradicionales. Dicho diseño factorial presenta una eficacia óptima, a pesar de tener un número de combinaciones más limitado entre otras cuestiones porque se han eliminado posibles errores en la implementación del cuestionario y se ha reducido el tiempo necesario para la obtención de sus resultados.

En este modelo los estímulos de las preferencias (como utilidades) de los encuestados difieren unos de otros, por lo que todos estos estímulos son evaluados por el encuestado de forma combinada y colectiva, a pesar de que sólo puntúe un número reducido. Con ello se puede calcular la utilidad estimada por el individuo respecto a los niveles que forman cada uno de los atributos a cuantificar en el bien inmueble, que nos sitúa en el aspecto clave para nuestro estudio.

\footnotetext{
9 El programa informático básicamente considera 3 aspectos: 1.- "Interviewing module": implementa directamente las respuestas de los entrevistados y las adopta a ellos; 2.- "Utility calculator": permite estimar las utilidades de cada nivel que conforma el atributo; 3.- "market simulator": establece la posibilidad de simular diferentes escenarios a mostrar al consumidor.
} 
Respecto al número de atributos (Green y Srinivasan, 1990) llegaron a 2 conclusiones:

- Si los atributos eran pocos ( $\leq 6)$, lo adecuado era utilizar el método del Análisis Conjunto de perfiles completos "Full profile”, ya que las estimaciones que proporciona son más precisas.

- Si el número de atributos se sitúa entre 6 y 9, se debe utilizar un modelo híbrido, dentro del cual se encuentra el "Adaptive Conjoint Analysis" (ACA).

Durante su desarrollo, el ACA explica más niveles de investigación al permitir mostrar a los encuestados más comparaciones por pares. Además, calcula la importancia relativa de cada atributo, obteniendo su nivel de utilidad real para el consumidor (valor de utilidad mayor - valor de utilidad menor), expresado como el porcentaje de la suma total de estos. Sin embargo, esta técnica presenta varias limitaciones, tal y como indica Rotaris (2002, pág. 107) "Uno dei limiti principali delle stime prodotte dall'ACA è rappresentato dal fatto che le utilità parziali standardizzate dei livelli di due attributi diversi non possono essere confrontate direttamente al fine di determinare quanto più utile sia un attributo rispecto all'altro. La procedura di standardizzazione delle stime delle utilità parziali e di eliminazione dei livelli inaccettabili rende, infatti, privo di significato tale confronto. L'ACA, però, non stima un parametro che quantifichi l'utilità percepita dall'intervistato in corrispondenza di qualunque valore venga assunto dall'attributo."

Se eligió ACA para este estudio para evitar la sobrecarga cognitiva que podría ocurrir en una comparación amplia de materiales de construcción para viviendas. En nuestro caso, que el encuestado no aceptase un material significa que no lo consideraba apropiado para la aplicación en pavimentos o revestimientos interiores de una estancia del uso residencial, bien fuese el baño, la cocina, el salón o el dormitorio, valorando mínimamente esta consideración respecto a la percepción de la estimación del valor de mercado de la vivienda. No obstante, se asume y sitúa entre los límites que marcan los oferentes y los demandantes para acotar dicha estimación. Antes de concluir con la evolución de esta técnica, se enuncia la forma estadística de la utilidad en el modelo básico ${ }^{10}$ del CA, siendo la función de utilidad general de la alternativa definida por:

"UTLij”: utilidad.

$$
U T L i j=\sum \underset{j=1}{\sum{ }_{j=1}^{L i} \underset{i=1}{M} x_{i j}+e i}
$$

“i = 1,....., M": número de atributos del producto.

“j = 1,......, Li”: número de niveles de cada atributo del producto.

"wij": coeficiente de regresión que expresa el peso otorgado por los encuestados, aportación del valor parcial o utilidad, con el j-ésimo nivel del i-ésimo atributo.

"xij": variable binaria (dummy) que se representa con el j-ésimo nivel del i-ésimo atributo.

"ei”: error asociado a la preferencia del i-ésimo producto, de relación tipo estadística y no matemática.

$\mathrm{M}$

El número de "estímulos" se obtiene de $\Pi$ Li, lo que significa que los productos para los que se hayan previsto múlti-

ples niveles ( $\mathrm{Li}=1$ ) no aumentan el número de productos posibles. Esta evolución metodológica conduce a que los atributos comparados en la n-ésima preferencia dependen de los atributos comparados en la (n-1) ésima preferencia asociada a la respuesta dada.

La originalidad del artículo es que se aplica en arquitectura la metodología de una técnica aplicativa híbrida del análisis conjunto, denominada análisis conjunto adaptativo, y se presenta una revisión de sus fundamentos teóricos y de software básicos desde un punto de vista de análisis cualitativo crítico, a menudo explicado brevemente en los escasos artículos de referencia existentes en España

\footnotetext{
10 Consultas realizadas para la utilización del programa ACA 5.1 System, SMRTV.4.20.2 de la Sawtooth Software Inc, consultado en www.sawtoothsoftware.com el 13122019.
}

ACE, 15 (43) CC BY-ND 3.0 ES | UPC Barcelona, España | Preferencias de la demanda sobre los materiales de construcción

del inmueble residencial: caso de estudio con el Análisis Conjunto Adaptativo. DOI: http://dx.doi.org/10.5821/ace.15.43.9245 
(Ferreira, 2010). Asimismo, el artículo pretende dar a conocer, a los profesionales del sector de la construcción y del ámbito inmobiliario, las bases conceptuales y metodológicas del Análisis Conjunto Adaptativo (ACA). Concretamente, presenta y describe la metodología de una técnica aplicativa híbrida del Análisis Conjunto, desarrollada para mejorar la complejidad del experimento al limitar el número de perfiles que se presenta al entrevistado. Asimismo, se reportan los primeros resultados de una investigación que ha tenido por objeto indagar, en la provincia de Alicante, hasta qué punto las técnicas de evaluación de la valoración estandarizada, y en concreto el análisis conjunto adaptativo, podrían ser utilizadas para tener en cuenta las preferencias y opiniones de los ciudadanos y los técnicos expertos en el sector de la construcción en la estimación de las tasas marginales de sustitución de la utilidad de los materiales de construcción de los inmuebles residenciales, para cuantificar los cambios de percepción de los individuos provocados por un cambio de elección de material. Desde el punto de vista operativo, la estimación de la importancia relativa de los atributos de los materiales de construcción evaluados, utilizando la técnica del ACA, muestra el gran impacto que la localización del material en la vivienda tiene sobre el comportamiento de elección de los consumidores.

El resto del artículo se estructura así: 1) primero se realiza una exposición sobre la naturaleza del análisis conjunto adaptativo, 2) a continuación se explicita la metodología y el caso de estudio abordado, y 3) enseguida se exponen los principales resultados y conclusiones en el marco de las preferencias de la demanda sobre los materiales de construcción del inmueble residencial a la luz de los resultados obtenidos.

\subsection{Metodología: Introducción al software ACA}

El paquete de software Adaptive Conjoint Analysis se utiliza para experimentos del AC y está presente en el aplicativo System, SMRTV.4.20.2 de la Sawtooth Software Inc. (1985-2010)11. El software del programa permite al encuestador realizar una entrevista interactiva con un ordenador portátil que gestiona la entrevista de forma automática. La entrevista está estructurada de manera inteligente, al personalizarse para cada entrevistado (las utilidades de los entrevistados se reevalúan a través del software en base a su elección), al finalizar la entrevista se dispondrá de las utilidades finales del entrevistado.

A continuación, se explica brevemente cómo se establecen las fases en el aplicativo del ACA:

1. Interviewing settings: de forma independiente, se recogen los datos de cada uno de los encuestados que han contestado toda la encuesta, verificándose una vez finalizada. 2. Utily run: el software permite calcular las utilidades para cada nivel de atributo presentado mediante el método ACA, a través del Ordinary Least Square (OLS), que utiliza para el cálculo del modelo de regresión de mínimos cuadrados, donde la variable dependiente está en escala de intervalo. 3.Market simulation ${ }^{12}$ : da lugar a la simulación de las alternativas del producto en base al estudio de los diferentes escenarios competitivos y permite al investigador definir un caso base que especifique las características del nuevo material propuesto para el bien vivienda. Para ello es necesario que indique los nuevos niveles de cada factor, obteniendo una simulación de la decisión de la demanda (como consumidor). Asimismo, le permite restringir la simulación a los encuestados que pertenezcan a segmentos

\footnotetext{
${ }^{11}$ En la investigación, se ha considerado el principal inconveniente de la utilización de este modelo, el cual permite estimar las utilidades parciales para cada nivel de cada atributo, pero no la utilidad de tales atributos.

${ }^{12}$ Es uno de los métodos más utilizados en los estudios del Conjoint Analysis. El simulador del programa convierte las utilidades parciales estimadas en simulaciones del comportamiento del cliente. Por ello, gracias a dichas simulaciones se pueden evaluar y establecer diversos escenarios hipotéticos que ayudan a mejorar el producto o servicio percibido por el consumidor.
}

ACE, 15 (43) CC BY-ND 3.0 ES | UPC Barcelona, España | Preferencias de la demanda sobre los materiales de construcción 
específicos del mercado, pudiendo asignar diferentes pesos a los diferentes grupos de consumidores. Finalmente, el investigador tiene la posibilidad de realizar un análisis de sensibilidad conjunta sobre los atributos individuales.

Paralelamente, resulta interesante mencionar que el cuestionario divide la entrevista esencialmente en 4 partes que explicamos a continuación y que serán ampliadas en el ejemplo del aplicativo con ACA:

- 10 Parte: Explicated Method. Combina 3 fases para obtener una primera estimación de las utilidades parciales de cada nivel. Fase 1: Unacceptables. El entrevistado debe indicar si alguno de los niveles que definen los atributos lo considera totalmente inaceptable, ya que, en este caso por tener asociado un valor por defecto de -9,999, dichos atributos no serán posteriormente utilizados en el test. Fase 2: Ratings and rankings levels. Permite establecer la jerarquía de priorización del grado de preferencia e importancia que el encuestado da a los atributos o las alternativas propuestas. En esta fase el ranking no se trata de un ejercicio de clasificación entre los atributos, sino del obtenido de la fase ranking precedente de cada atributo presentado al entrevistado. Al hacer esto, se usa una escala Likert de 1 a 5, donde 1 identifica la importancia menor (no deseable/preferible) y 5 (extremadamente deseable /preferible). Fase 3: Importance scale. Mediante una escala Likert de 1 a 5, permite identificar su mayor grado de la preferencia de los atributos después de que hayan sido presentados el mejor y el peor nivel al entrevistado, obtenido de la fase de ranking precedente, de cada uno de los atributos enfrentados.

- 2a Parte: Pairs. Al encuestado se le presentan 2 perfiles del producto a la vez (método Paired Comparison Scale), formados por la comparación de dos o más atributos de todas las posibles combinaciones de niveles, y éste debe elegir 1 de los 2 perfiles. Así, el encuestado no puntúa los perfiles descritos por todos los atributos y niveles, sino que sólo lo hace de aquellos que en los que en sus precedentes respuestas consideró más importantes y preferidos.

- 3a Parte: Calibration concepts. El software presenta diversas opciones del producto, utilizando los niveles y atributos considerados como relevantes. El encuestado deberá indicar la probabilidad de adquirir la opción presentada en la entrevista, en base a las preferencias puntuadas anteriormente. La entrevista es percibida de manera personalizada y sirve principalmente al sistema para calibrar las utilidades previamente calculadas.

- 4a Parte: Randomization. Esta parte añade un error al cálculo previo de las utilidades para evaluar la fiabilidad de las respuestas facilitadas por el entrevistado.

\subsection{Caso de estudio con el aplicativo ACA}

A continuación, se presenta (Tabla 2 y Tabla 3) un ejemplo de aplicación simple del software ACA, que permitirá aclarar las partes descritas en el punto anterior de una manera más concreta.

Básicamente, se identifican para esta investigación 8 atributos, tales como: baño pavimento, baño revestimiento, cocina pavimento, cocina revestimiento, salón pavimento, salón revestimiento, dormitorio pavimento y dormitorio revestimiento. El ejemplo en cuestión se refiere a evaluar y medir el grado de bienestar en el uso residencial, que reporta tanto a usuarios como a técnicos en edificación y expertos en la materia, un determinado grupo de atributos asociados a la percepción de los materiales de construcción, para su aplicación en pavimentos y revestimientos interiores. Como se muestra en la Tabla 2 y Tabla 3 cada uno de estos atributos tiene varios niveles.

ACE, 15 (43) CC BY-ND 3.0 ES | UPC Barcelona, España | Preferencias de la demanda sobre los materiales de construcción

del inmueble residencial: caso de estudio con el Análisis Conjunto Adaptativo. DOI: http://dx.doi.org/10.5821/ace.15.43.9245 
Tabla 2. Atributos y niveles relativos a la estimación estandarizada de la utilidad de los materiales de construcción en el uso residencial para zonas húmedas

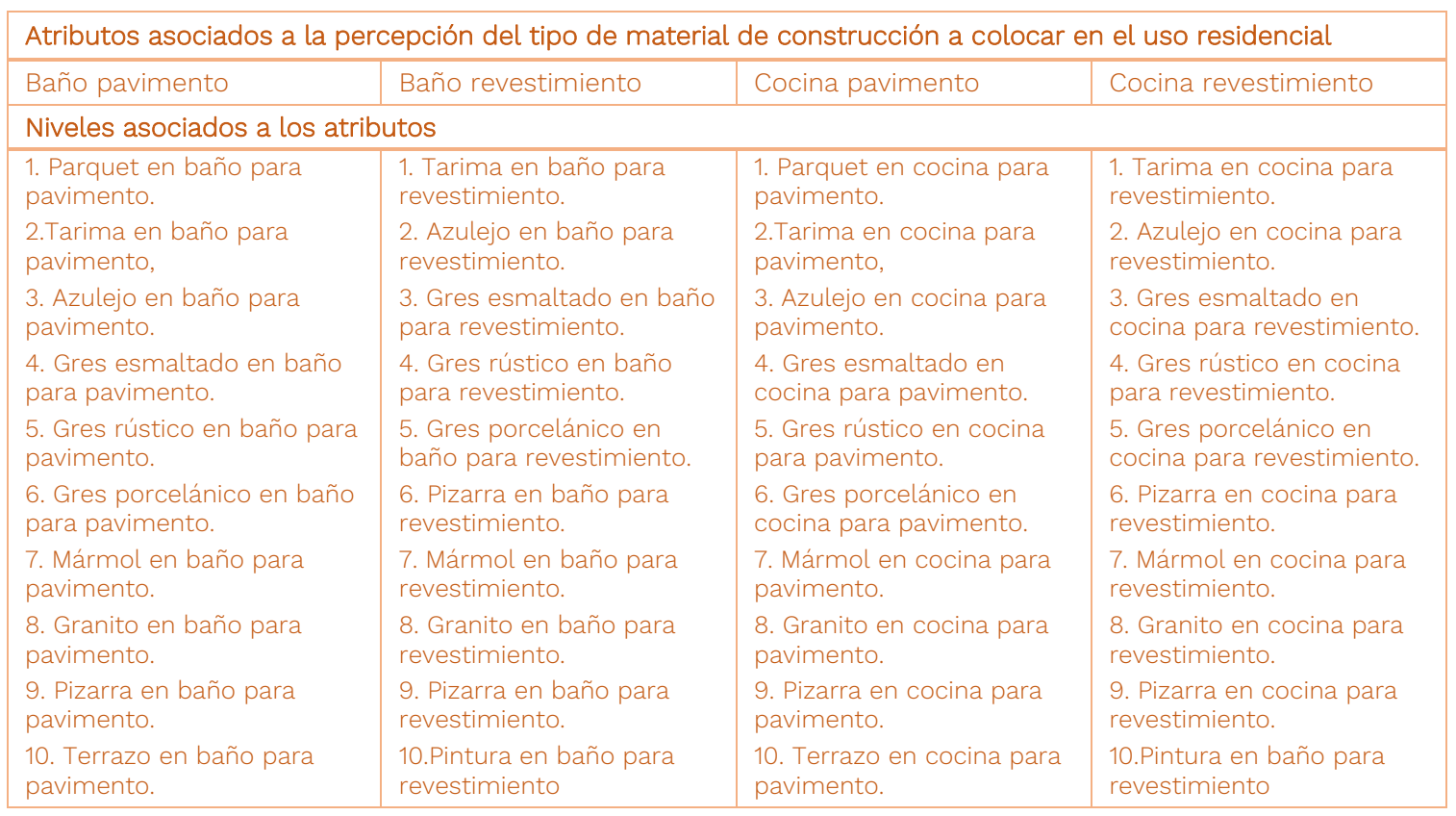

Fuente: Elaboración propia

Tabla 3. Atributos y niveles relativos a la estimación estandarizada de la utilidad de los materiales de construcción en el uso residencial para resto de estancias

\begin{tabular}{|c|c|c|c|}
\hline Salón pavimento & Salón revestimiento & Dormitorio pavimento & Dormitorio revestimiento \\
\hline \multicolumn{4}{|c|}{ Niveles asociados a los atributos } \\
\hline $\begin{array}{l}\text { 1. Parquet en salón para } \\
\text { pavimento. } \\
\text { 2.Tarima en salón para } \\
\text { pavimento, } \\
\text { 3. Azulejo en salón para } \\
\text { pavimento. } \\
\text { 4. Gres esmaltado en } \\
\text { salón para pavimento. } \\
\text { 5. Gres rústico en salón } \\
\text { para pavimento. } \\
\text { 6. Gres porcelánico en } \\
\text { salón para pavimento. } \\
\text { 7. Mármol en salón para } \\
\text { pavimento. } \\
\text { 8. Granito en salón para } \\
\text { pavimento. } \\
\text { 9. Pizarra en salón para } \\
\text { pavimento. } \\
\text { 10. Terrazo en salón para } \\
\text { pavimento. }\end{array}$ & $\begin{array}{l}\text { 1. Tarima en salón para } \\
\text { revestimiento. } \\
\text { 2. Azulejo en salón para } \\
\text { revestimiento. } \\
\text { 3. Gres esmaltado en } \\
\text { salón para revestimiento. } \\
\text { 4. Gres rústico en salón } \\
\text { para revestimiento. } \\
\text { 5. Gres porcelánico en } \\
\text { salón para revestimiento. } \\
\text { 6. Pizarra en salón para } \\
\text { revestimiento. } \\
\text { 7. Mármol en salón para } \\
\text { revestimiento. } \\
\text { 8. Granito en salón para } \\
\text { revestimiento. } \\
\text { 9. Pizarra en salón para } \\
\text { revestimiento. } \\
\text { 10.Pintura en salón para } \\
\text { revestimiento } \\
\text { 11. Vidrio en salón para } \\
\text { revestimiento. } \\
\text { 12. Papel en salón para } \\
\text { revestimiento. }\end{array}$ & $\begin{array}{l}\text { 1. Parquet en dormitorio } \\
\text { para pavimento. } \\
\text { 2.Tarima en dormitorio } \\
\text { para pavimento, } \\
\text { 3. Azulejo en dormitorio } \\
\text { para pavimento. } \\
\text { 4. Gres esmaltado en } \\
\text { dormitorio para } \\
\text { pavimento. } \\
\text { 5. Gres rústico en } \\
\text { dormitorio para } \\
\text { pavimento. } \\
\text { 6. Gres porcelánico en } \\
\text { dormitorio para } \\
\text { pavimento. } \\
\text { 7. Mármol en dormitorio } \\
\text { para pavimento. } \\
\text { 8. Granito en dormitorio } \\
\text { para pavimento. } \\
\text { 9. Pizarra en dormitorio } \\
\text { para pavimento. } \\
\text { 10. Terrazo en dormitorio } \\
\text { para pavimento. }\end{array}$ & $\begin{array}{l}\text { 1. Tarima en dormitorio para } \\
\text { revestimiento. } \\
\text { 2. Azulejo en dormitorio para } \\
\text { revestimiento. } \\
\text { 3. Gres esmaltado en } \\
\text { dormitorio para } \\
\text { revestimiento. } \\
\text { 4. Gres rústico en dormitorio } \\
\text { para revestimiento. } \\
\text { 5. Gres porcelánico en } \\
\text { dormitorio para } \\
\text { revestimiento. } \\
\text { 6. Pizarra en dormitorio para } \\
\text { revestimiento. } \\
\text { 7. Mármol en dormitorio para } \\
\text { revestimiento. } \\
\text { 8. Granito en dormitorio para } \\
\text { revestimiento. } \\
\text { 9. Pizarra en dormitorio para } \\
\text { revestimiento. } \\
\text { 10.Pintura en dormitorio para } \\
\text { revestimiento } \\
\text { 11. Vidrio en dormitorio para } \\
\text { revestimiento. } \\
\text { 12.Papel en dormitorio para } \\
\text { revestimiento }\end{array}$ \\
\hline
\end{tabular}

Fuente: Elaboración propia

ACE, 15 (43) CC BY-ND 3.0 ES | UPC Barcelona, España $\mid$ Preferencias de la demanda sobre los materiales de construcción

del inmueble residencial: caso de estudio con el Análisis Conjunto Adaptativo. DOI: http://dx.doi.org/10.5821/ace.15.43.9245 
Para obtener una valoración realista, tanto los atributos como sus niveles deben ser fácilmente comunicables y presentar un rango apropiado, ya que ante un rango excesivamente amplio existiría la posibilidad de que un técnico (o experto) no esté dispuesto a cambiar un nivel por otro. Por ello, este planteamiento considera que estos atributos deben permitir al método del CA combinar los modelos conjuntos de componentes parciales de la utilidad total con los auto-explicados obtenidos del diseño factorial fraccional.

Veamos ahora las fases del cuestionario presentado al encuestado:

1. La primera fase del cuestionario es necesaria para descartar la no deseabilidad/preferencia de los materiales de construcción en función de su ubicación y colocación para un uso residencial. Posteriormente, la 2 a fase de la entrevista tiene el objetivo de identificar los atributos más importantes para el sujeto, es decir, de poder detectar tanto los materiales muy mal y muy bien percibidos, como la deseabilidad/preferencia en pavimentos y revestimientos de las estancias: baño, cocina, salón y dormitorio. Respecto a los niveles decir que se optará por eliminar de la entrevista final a los casos de materiales muy mal y mal percibidos, si éstos guardan coherencia con los resultados obtenidos una vez analizados los datos de todas las entrevistas. A continuación, cada encuestado ordena las utilidades en una escala Likert (1 a 5), de acuerdo a las preferencias de los distintos niveles de los atributos presentados durante la encuesta, utilizando para ello la modalidad de escalamiento rating. El objetivo de la 3a parte de la entrevista es graduar y ordenar las preferencias de los encuestados sobre los materiales de construcción que, como alternativas de los productos objeto de análisis, se muestran para una ubicación y aplicación concreta de la vivienda, detectándose el orden de las preferencias, mejores y peores, de los niveles de cada uno de los atributos mostrados al encuestado y comprobando si existe algún nivel con baja puntuación que deba ser eliminado.

El siguiente paso de la entrevista será pasar del Full-profile a la comparación de parejas. Para ello se elabora un conjunto de descripciones del uso residencial con todos los atributos escogidos, pero sólo un nivel por atributo. En este punto se pide al encuestado que indique cuál de las dos alternativas mostradas prefiere. A pesar de que el encuestado se decline sólo por un atributo, se debe considerar que el software se basará en los datos obtenidos exclusivamente de la 3a parte de la entrevista, actualizando dichos datos conforme avanza ésta, ya que a los atributos inaceptables de la $2 \underline{a}$ parte les da un valor arbitrario negativo. En líneas generales, la alternativa es elegida aleatoriamente por el ordenador mediante un modelo de regresión basado en los mínimos cuadrados (OLS Ordinary Least Squares), describiendo el material a valorar teniendo en cuenta sus características (atributos), subdivididas a través de los diferentes niveles preestablecidos. Es más, al asumir la variable dependiente la naturaleza de intervalo, esta técnica permite conocer los errores estandarizados para los parámetros estimados

En virtud de lo anterior, el encuestado debe indicar su preferencia por un material u otro para el uso residencial, considerando la ubicación y colocación específica del mismo. Para ello, cuantificará su preferencia apoyándose en una escala Likert de (1 a 5). En la 4a parte de la entrevista se dan 2 casuísticas: 6 preguntas formadas por la combinación de 2 niveles Importance y otras 6 preguntas formadas por más de 2 niveles Pairs. Con ello, se obtienen las utilidades individuales de cada encuestado a través de una serie de comparaciones pareadas. En la práctica, las propuestas a realizar para la baremación nos dan un valor total de utilidad simple, en base a una primera estimación de las utilidades parciales de cada uno de los niveles. A partir de este punto, aumentará la dificultad de elección de preferencia que el encuestado debe tomar, al adaptarse a él la entrevista hasta el punto donde cada atributo y cada nivel vienen presentados con igual frecuencia, siendo por tanto el objetivo de esta $4^{a}$ parte el escalar los valores con un rango de variación par.

ACE, 15 (43) CC BY-ND 3.0 ES | UPC Barcelona, España | Preferencias de la demanda sobre los materiales de construcción del inmueble residencial: caso de estudio con el Análisis Conjunto Adaptativo. DOI: http://dx.doi.org/10.5821/ace.15.43.9245 
La entrevista finaliza con la 5a parte, denominada Calibration concepts, en la que se facilita al encuestado una posible solución de materiales de construcción para pavimentos y revestimientos para uso residencial. En esta parte se solicita al encuestado la preferencia de utilización de esos materiales propuestos, aleatoriamente, para esas aplicaciones y localizaciones concretas. En este punto, la respuesta del entrevistado permite calibrar las utilidades que el software ha incluido previamente, para así poder evaluar la fiabilidad de las respuestas del encuestado, es decir, se representará la estructura de preferencia del consumidor que incluye el proceso de segmentación, en el cual se separan las componentes parciales estimadas de la utilidad total para obtener agrupaciones de encuestados que presenten similitud en sus preferencias. Es importante aclarar que, en esta 5a fase, el software establece los coeficientes de regresión simple de tipo Bayesiano, los cuales le permiten determinar los pesos que cuantifican los atributos y niveles que constituyen la elección de los materiales de construcción para pavimentos y revestimientos de cualquier uso residencial. En virtud de lo anterior, las posibles combinaciones de los atributos y sus correspondientes niveles ubicados en el contexto de elección, representarán el diseño experimental del ACA.

\subsubsection{Análisis de la parte experimental con el file ACD}

Una vez recogidos los datos y para asegurar que las utilidades están constituidas por los 8 atributos más relevantes para el entrevistado, el sistema recupera automáticamente los valores de utilidad media mediante el file $A C D^{13}$. Todo ello, para calcular la importancia de los atributos e identificar los 8 atributos más importantes para permitir posteriormente interpretar los resultados. Si bien no es el objeto de este artículo realizar una explicación técnica de dicho file, es necesario realizar una somera presentación del mimo debido a su relativo desconocimiento en la aplicación práctica en la disciplina de la arquitectura. Siendo el objetivo evidenciar cómo el programa distingue las fases en el cuestionario para obtener el peso de las utilidades, la Tabla 3 muestra un ejemplo de los resultados obtenidos de una de las entrevistas realizadas en la investigación. Aquí, el software opera un 1o orden considerando los atributos definidos con sus respectivos niveles, es decir, el número de la entrevista (del 1 al 209), el número de pairs (6), el número de atributos (8), el número de niveles estudiados (82).

La Parte A (superior derecha de la Tabla 4) muestra el orden de preferencia en función de los 8 atributos. Si analizamos, por ejemplo, la tercera fila del bloque A del file ACD, se da la puntuación para el atributo 3 planteado (cocina pavimento). Lo importante aquí es la posición del valor y no el valor en sí, ya que se deben leer de manera invertida los 10 valores que definen el atributo 3, es decir, en la Tabla 4 deberemos leer (-1, -1, -1, -1, 4, 4, 4, -1, 3, 3). Para su interpretación, recordemos que los 10 valores eran equivalentes a: 1 . Parquet en cocina para pavimento, 2. Tarima en cocina para pavimento, 3. Azulejo en cocina para pavimento, 4. Gres esmaltado en cocina para pavimento, 5. Gres rústico en cocina para pavimento, 6. Gres porcelánico en cocina para pavimento, 7. Mármol en cocina para pavimento, 8. Granito en cocina para pavimento, 9. Pizarra en cocina para pavimento, 10. Terrazo en cocina para pavimento.

Por tanto, en la Parte $A$ sólo son aceptados los materiales gres rústico, gres porcelánico, mármol, pizarra y terrazo. Entre ellos, obtienen una mayor puntuación (valor 4) el gres rústico, gres porcelánico y mármol, frente a la pizarra y terrazo con menor puntuación (valor 3). Luego en esta primera fase se establece el orden de preferencia de todos los atributos presentados al encuestado

La Parte $B$ (superior izquierda de la Tabla 4) muestra la importancia relativa de cada uno de los atributos presentados al encuestado. En relación con el resto de atributos, el software determina los

\footnotetext{
${ }^{13}$ En Italia el trabajo de Arboretti (2003) y en Berlin el trabajo de Herrmann, Schmidt y Huber, (2003) constituyen unas referencias de primer orden al brindar el panorama de los primeros estudios de desarrollo del software de esta metodología.
}

ACE, 15 (43) CC BY-ND 3.0 ES | UPC Barcelona, España Preferencias de la demanda sobre los materiales de construcción 
productos que se han considerado aceptables y, a su vez, cuáles tienen más importancia para el encuestado. Si analizamos, por ejemplo, la primera columna del bloque B del file ACD corresponde a los valores 4, 12, 24, 32, 41, 59, 62 y 80, que equivalen a los diferentes niveles de los atributos (ver Tablas 1 y 2). Para su interpretación, recordemos que estos 8 valores eran equivalentes a: 4 . Gres esmaltado en baño para pavimento, 12. Azulejo en baño para revestimiento, 24. Gres esmaltado en cocina para pavimento, 32. Azulejo en cocina para revestimiento, 41. Tarima en salón para pavimento, 59. Pintura en salón para revestimiento, 62. Tarima en salón para pavimento, 80. Vidrio en dormitorio para revestimiento. Por tanto, el atributo que adquiere mayor importancia es el del baño para pavimento, siendo el gres esmaltado en baño el más destacado en este nivel (valor 4), mientras que la importancia menor la adquiere el atributo dormitorio para revestimiento, en el que destaca el vidrio por su baja puntuación (valor 80).

Tabla 4. Exposición del file ACD

\begin{tabular}{|c|c|c|c|c|c|c|c|c|c|}
\hline Parte B & \multicolumn{9}{|c|}{ Parte A } \\
\hline 4 & 4 & -1 & -1 & 4 & -1 & -1 & -1 & 0 & 0 \\
\hline 12 & 5 & 4 & 2 & 3 & -1 & -1 & 0 & 0 & 0 \\
\hline 24 & 5 & 3 & -1 & 4 & -1 & -1 & -1 & 0 & 0 \\
\hline 32 & 5 & 4 & 2 & 3 & -1 & -1 & 3 & 0 & 0 \\
\hline \begin{tabular}{l|l}
41 & 48 \\
\end{tabular} & 4 & 4 & -1 & 4 & 3 & -1 & 0 & 0 & 0 \\
\hline 59 & 5 & -1 & 2 & 3 & 3 & 5 & 5 & 0 & 0 \\
\hline 62 & 4 & 4 & -1 & 3 & 4 & -1 & 0 & 0 & 0 \\
\hline 80 & 5 & -1 & 2 & 3 & 3 & 5 & -1 & 0 & 0 \\
\hline \multicolumn{10}{|l|}{ Parte C } \\
\hline-18 & -22 & 13 & 24 & 0 & 0 & 5 & 0 & 0 & 1 \\
\hline-12 & -78 & 13 & 80 & 0 & 0 & 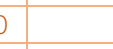 & 0 & 0 & 3 \\
\hline-74 & -37 & 72 & 33 & 0 & 0 & 5 & 0 & 0 & 3 \\
\hline-54 & -35 & 57 & 36 & 0 & 0 & D & 0 & 0 & 4 \\
\hline-53 & -66 & 54 & 68 & 0 & 0 & 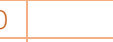 & 0 & 0 & 3 \\
\hline-45 & -71 & 44 & 68 & 0 & 0 & 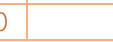 & 0 & 0 & 2 \\
\hline \multicolumn{10}{|l|}{ Parte E } \\
\hline$-9,999$ & $-9,999$ & $-9,999$ & 0,000 & 0,000 & 0,000 & $-9,999$ & $-9,999$ & $-9,999$ & $-9,999$ \\
\hline$-9,999$ & 2,736 & $-1,303$ & $-1,113$ & $-1,113$ & 0,835 & $-9,999$ & 1,071 & $-9,999$ & $-1,113$ \\
\hline$-2,338$ & $-2,101$ & $-9,999$ & 1,322 & 1,559 & 1,559 & $-9,999$ & $-9,999$ & $-9,999$ & $-9,999$ \\
\hline$-9,999$ & 2,505 & $-1,392$ & $-1,392$ & $-1,515$ & 0,680 & 0,557 & $-9,999$ & $-9,999$ & 0,557 \\
\hline 0,390 & 0,390 & 0,390 & $-9,999$ & 0,266 & 0,513 & 0,390 & $-2,728$ & $-9,999$ & 0,390 \\
\hline$-1,039$ & $-1,03$ & $-9,999$ & $-1,088$ & $-1,113$ & $-1,039$ & 0,260 & 0,260 & 2,857 & $-1,039$ \\
\hline 2,857 & 1,169 & 1,169 & 1,169 & $-9,999$ & $-1,948$ & $-1,998$ & 1,095 & 1,169 & $-9,999$ \\
\hline 1,292 & $-0,144$ & $-9,999$ & $-9,999$ & $-1,443$ & $-1,443$ & $-1,443$ & $-0,192$ & $-0,144$ & 2,501 \\
\hline$-9,999$ & 2,453 & - & - & - & - & - & - & - & - \\
\hline$-9,999$ & $-9,999$ & $-9,999$ & 0,000 & 0,000 & 0,000 & $-9,999$ & $-9,999$ & $-9,999$ & $-9,999$ \\
\hline$-9,999$ & 2,857 & $-1,143$ & $-1,143$ & $-1,143$ & 0,857 & $-9,999$ & 0,857 & $-9,999$ & $-1,143$ \\
\hline$-2,400$ & $-2,400$ & $-9,999$ & 1,600 & 1,600 & 1,600 & $-9,999$ & $-9,999$ & $-9,999$ & $-9,999$ \\
\hline$-9,999$ & 2,571 & $-1,429$ & $-1,429$ & $-1,429$ & 0,571 & 0,571 & $-9,999$ & $-9,999$ & 0,571 \\
\hline 0,400 & 0,400 & $-9,999$ & 0,400 & 0,400 & 0,400 & 0,400 & $-2,800$ & $-9,999$ & 0,400 \\
\hline$-1,067$ & $-9,999$ & $-1,067$ & $-1,067$ & $-1,067$ & 0,267 & $-1,067$ & 0,267 & 2,933 & $-1,067$ \\
\hline 2,933 & 1,200 & 1,200 & $-9,999$ & $-2,000$ & $-2,000$ & $-2,000$ & 1,200 & 1,200 & $-9,999$ \\
\hline 1,200 & $-0,148$ & $-9,999$ & $-1,481$ & $-1,481$ & $-1,481$ & $-0,148$ & $-0,148$ & $-0,148$ & 2,519 \\
\hline$-9,999$ & 2,519 & - & - & - & - & & - & - & - \\
\hline 0,000 & 0,000 & 0,000 & 0,000 & 0,000 & 0,000 & 0,000 & 0,000 & 0,000 & 0,000 \\
\hline 0,000 & $-0,167$ & $-0,667$ & 0,000 & 0,000 & 0,000 & 0,000 & 0,833 & 0,000 & 0,000 \\
\hline 0,000 & 0,833 & 0,000 & $-0,833$ & 0,000 & 0,000 & 0,000 & 0,000 & 0,000 & 0,000 \\
\hline 0,000 & 0,000 & 0,000 & 0,000 & $-0,435$ & 0,435 & 0,000 & 0,000 & 0,000 & 0,000 \\
\hline 0,000 & 0,000 & 0,000 & $-0,435$ & 0,435 & 0,000 & 0,000 & 0,000 & 0,000 & 0,000 \\
\hline 0,000 & 0,000 & $-0,174$ & $-0,261$ & 0,000 & 0,000 & 0,435 & 0,000 & 0,000 & 0,000 \\
\hline 0,000 & 0,000 & 0,000 & 0,000 & 0,000 & $-0,174$ & 0,000 & $-0,261$ & 0,000 & 0,000 \\
\hline 0,435 & 0,000 & 0,000 & 0,000 & 0,000 & 0,000 & 0,000 & $-0,167$ & 0,000 & 0,167 \\
\hline
\end{tabular}




\section{ACE Architecture, City and Environment}

\begin{tabular}{|r|r|r|r|r|r|r|r|r|}
\hline Parte D & \multicolumn{1}{|c|}{ Utilidad } \\
\hline 6 & 6 & - & - & - & - & - & - & 30 \\
\hline 21 & 13 & 74 & 33 & 51 & 65 & $-9,463$ & 0,000 & 80 \\
\hline 24 & 12 & 80 & 32 & 59 & 62 & 13,090 & 0,000 & 50 \\
\hline 21 & 13 & 74 & 32 & 59 & 62 & 1,448 & 0,000 & 50 \\
\hline 24 & 12 & 74 & 33 & 51 & 65 & $-1,764$ & 0,000 & 60 \\
\hline 21 & 13 & 80 & 32 & 59 & 62 & 5,392 & 0,000 & 70 \\
\hline 24 & 12 & 80 & 33 & 51 & 65 & 2,180 & 0,000 & - \\
\hline 0,124 & 0,096 & 0,000 & 0,868 & - & - & - & -- & \\
\hline
\end{tabular}

Fuente : Elaboración propia

La Parte C (central de la Tabla 4) muestra el paso de Full Pro-file to Pairs Comparison, es decir, la confrontación en parejas. Si analizamos como ejemplo la cuarta fila, con los valores -54, -35, 57, 36, 0, 0, 0, 0, 0, 0 y 4, encontramos que, de manera distinta a las demás partes, tan solo los cuatro primeros corresponden a los diferentes niveles de atributos (ver Tablas 2 y 3), que equivalen a: -54. Gres esmaltado en salón para pavimento, -35. Gres rústico en pavimento cocina, 57. Mármol en salón para pavimento, 36. Gres porcelánico en cocina para pavimento. Los seis siguientes ceros indican que se han realizado 6 comparaciones de pareja y el último valor (4) se corresponde con la escala Likert. Por tanto, en este ejemplo el encuestado considera muy deseable/preferible la opción que contiene el mármol en salón para pavimento y el gres porcelánico en cocina para pavimento, frente a la opción del gres esmaltado en salón para pavimento y el gres rústico en cocina para pavimento. Por otro lado, se aclara que en esta parte $\mathrm{C}$, antes de comenzar a actualizar las estimaciones, se debe especificar qué valores asumen los elementos descritos anteriormente, en relación con el ejemplo que acabamos de mostrar para permitir al software ACA calcula las utilidades, empleando el Modelo de Regresión de los Mínimos Cuadrados o Ordinary Least Squares, por ser el que contiene el aplicativo de este programa.

La Parte E (central de la Tabla 4) se obtiene una vez finalizadas las partes A y B, al contar con los datos necesarios para que el software determine el primer conjunto de utilidades. Para ello, se calcula la media de los valores adjudicados a cada atributo (de 0 a 5 en nuestra investigación), para posteriormente situar cada valor respecto del cero: -3, -2, -1, 0, 1, 2, 3. Luego en esta segunda fase se determina la importancia relativa de las estimaciones iniciales de cada atributo presentado al encuestado. Esta Parte E se ejecuta mediante el desarrollo del algoritmo planteado por el software y utilizado para determinar las estimaciones iniciales de utilidad. Para ello, se llevan a cabo 4 pasos:

- Paso 1: Se invierte el orden de los valores.

- Paso 2: Se obtiene la media de las puntuaciones de los valores de cada atributo.

- Paso 3: Se re-escalan los valores en un rango de variación 1.

- Paso 4: Se establecen las utilidades relativas de los atributos.

La Parte D (inferior de la Tabla 4) muestra la importancia relativa de cada atributo, calculando la diferencia entre su mayor y su menor valor de utilidad. En el ejemplo en cuestión, con referencia al bloque D, los números 6 y 6 representan respectivamente: el número de conceptos de calibración presentados (estas son las seis líneas); el número de niveles de los conceptos de calibración presentados. Asimismo, decir que la primera línea, por ejemplo, se lee así: "¿Cuánto le satisface esta elección de materiales de construcción para pavimentos y revestimientos de un uso residencial? "La respuesta corresponde al último valor de la fila y en nuestro caso sería de 30\%. Los valores restantes de la fila $(74,33,51$ y 65$)$ se refieren a las utilidades iniciales del perfil propuesto, mientras que el valor -9,463 corresponde a la suma de utilidades derivadas de comparaciones por pares. Para determinar los valores de la intersección y los dos coeficientes, el software del ACA utiliza una formulación de la probabilidad de compra, como una función que depende del peso de los encuestados. Así, una vez que se han obtenido los valores (0,124, 0,096, 0,000 y 0,868), el software continúa con el cálculo de utilidades finales, que están contenidas en la extensión de archivo. UTL.

ACE, 15 (43) CC BY-ND 3.0 ES I UPC Barcelona, España Preferencias de la demanda sobre los materiales de construcción 
De ahí que, los rangos de los atributos se expresan como un porcentaje de la suma total de los rangos, siendo el resultado un indicador de la importancia relativa de cada atributo. Una vez obtenidos los resultados, se considera interesante realizar un análisis de los valores con porcentajes inaceptables. Por otro lado, este primer análisis de los resultados del ACA contempla la no deseabilidad/preferencia tanto en los 8 atributos como en sus distintos niveles, en función de su ubicación en zonas húmedas (Baño y Cocina) o zonas resto de estancias (Salón y Dormitorio) y, de su colocación (en pavimentos o en revestimientos) en la vivienda.

\section{Resultados del caso de estudio con el aplicativo ACA}

La Tabla 5 recoge y analiza cada uno de los materiales de construcción de la vivienda con valores porcentuales inaceptables, contemplando la no deseabilidad/preferencia tanto en los 8 atributos como en sus distintos niveles, en función de su ubicación y de su colocación en la vivienda. Al ser datos métricos, se debe de considerar que cuanto mayor y más próximo a 100 sea el valor, más inaceptado será el nivel por el entrevistado. Los datos mostrados en esta tabla resultan útiles, ya que eliminan un atributo o modifican su nivel con el fin de provocar un cambio en el valor del material percibido por el consumidor. Lo que es bastante sorprendente en los resultados de los datos es el hecho de que la diversidad de participantes, no tuvo un impacto importante para su aceptación. Más allá del hecho, que se observa que los encuestados sólo aceptan aquellos materiales que están ubicados y colocados correctamente. Asinino, en esta Tabla 5 respecto a los pavimentos, la percepción adecuada de la madera (parquet y tarima) en salón y dormitorio, hace que menos del 8\% de los encuestados lo consideren inaceptable. Respecto a los revestimientos, la pintura se percibe como muy adecuada en salón y dormitorio, obteniendo el 100\% de aceptación de los encuestados (0\% de rechazo). En el caso de la madera, sus puntuaciones se deben a su calidez y a la idea social de relacionarla a un mayor nivel adquisitivo, mientras que, en el caso de la pintura, éstas se deben a la diversidad de terminaciones que ofrece y a su bajo coste económico.

Tabla 5. Porcentaje de inaceptables con la metodología ACA

\begin{tabular}{|c|c|c|c|c|c|c|c|c|c|}
\hline \multirow{2}{*}{ Materiales } & \multicolumn{4}{|c|}{ Pavimento } & \multirow{2}{*}{ Materiales } & \multicolumn{4}{|c|}{ Revestimiento } \\
\hline & Baño & Cocina & Salón & Dormitorio & & Baño & Cocina & Salón & Dormitorio \\
\hline Parquet & 62,20 & 66,03 & 7,66 & 6,22 & Tarima & 62,68 & 72,73 & 42,11 & 44,02 \\
\hline Tarima & 60,77 & 64,59 & 6,70 & 7,18 & Azulejo & 31,58 & 25,36 & 78,47 & 80,86 \\
\hline Azulejo & 68,42 & 69,86 & 98,56 & 97,61 & $\begin{array}{l}\text { Gres } \\
\text { esmaltado }\end{array}$ & 38,76 & 37,80 & 66,51 & 68,90 \\
\hline $\begin{array}{l}\text { Gres } \\
\text { esmaltado }\end{array}$ & 43,06 & 38,76 & 66,99 & 70,33 & $\begin{array}{l}\text { Gres } \\
\text { rústico }\end{array}$ & 45,93 & 33,01 & 62,68 & 67,46 \\
\hline $\begin{array}{l}\text { Gres } \\
\text { porcelánico }\end{array}$ & 27,27 & 30,14 & 48,80 & 55,50 & $\begin{array}{l}\text { Gres } \\
\text { porcelánico }\end{array}$ & 26,79 & 25,84 & 58,37 & 62,68 \\
\hline $\begin{array}{l}\text { Gres } \\
\text { rústico }\end{array}$ & 46,41 & 24,88 & 52,63 & 53,11 & Mármol & 43,06 & 63,64 & 55,50 & 63,16 \\
\hline Mármol & 46,89 & 64,59 & 30,62 & 32,54 & Granito & 61,72 & 59,33 & 62,20 & 66,99 \\
\hline Granito & 60,29 & 61,72 & 60,29 & 63,64 & Pizarra & 52,15 & 57,42 & 52,63 & 58,37 \\
\hline Pizarra & 53,59 & 55,50 & 61,24 & 64,59 & Pintura & 44,98 & 40,67 & 0,00 & 0,00 \\
\hline Terrazo & 67,94 & 57,42 & 52,15 & 54,55 & Vidrio & 56,94 & 59,81 & 42,11 & 51,67 \\
\hline- & - & - & - & - & Papel & - & - & 21,05 & 18,66 \\
\hline
\end{tabular}

Fuente : Elaboración propia 
Los resultados obtenidos de la Tabla 5 indican que, en pavimentos, sólo los materiales versátiles (gres esmaltado, gres rústico, gres porcelánico para baño y cocina y, el mármol (en baño, salón y dormitorio) son deseables para más del 50\% de los encuestados. En revestimientos, para cerca del $50 \%$ de los encuestados tan sólo son deseables la pintura, el papel y el vidrio (en salón y dormitorio).

Por otro lado, la Tabla 6 analiza los resultados de la estimación estandarizada de la utilidad de los materiales de construcción de la vivienda, para las aplicaciones de pavimento y revestimiento en baño y cocina y, en salón y dormitorio, alcanzados con la técnica del ACA, estableciendo la utilidad para cada nivel de atributo analizado y reflejando el grado de preferencia que tiene para los encuestados/as en el uso residencial.

Tabla 6. Resumen de los valores de utilidad medios con la metodología ACA

\begin{tabular}{|c|c|c|c|c|c|c|c|c|c|}
\hline \multirow{2}{*}{ Materiales } & \multicolumn{4}{|c|}{ Pavimento } & \multirow{2}{*}{ Materiales } & \multicolumn{4}{|c|}{ Revestimiento } \\
\hline & Baño & Cocina & Salón & Dormitorio & & Baño & Cocina & Salón & Dormitorio \\
\hline Parquet & -9.87 & -11.00 & 39,25 & 44,41 & Tarima & $-16,88$ & $-24,43$ & $-5,41$ & $-7,59$ \\
\hline Tarima & 9.08 & -9.29 & 42,92 & 41,16 & Azulejo & 10,22 & 17,70 & $-21,03$ & $-18,47$ \\
\hline Azulejo & 11.02 & -10.64 & $-27,83$ & $-26,65$ & $\begin{array}{l}\text { Gres } \\
\text { esmaltado }\end{array}$ & 5,76 & 6,41 & $-18,24$ & $-16,74$ \\
\hline $\begin{array}{l}\text { Gres } \\
\text { esmaltado }\end{array}$ & 4.16 & 7.22 & $-16,70$ & $-16,23$ & $\begin{array}{l}\text { Gres } \\
\text { rústico }\end{array}$ & $-1,57$ & 13,62 & $-17,52$ & $-16,21$ \\
\hline $\begin{array}{l}\text { Gres } \\
\text { porcelánico }\end{array}$ & $\begin{array}{r}23.7 \\
6 \\
\end{array}$ & 29.34 & $-7,63$ & $-9,75$ & $\begin{array}{l}\text { Gres } \\
\text { porcelánico }\end{array}$ & 19,47 & 28,93 & $-16,57$ & $-15,47$ \\
\hline Gres rústico & 4.06 & 21.92 & $-7,67$ & $-8,79$ & Mármol & 8,87 & $-13,16$ & $-12,18$ & $-15,30$ \\
\hline Mármol & 9.78 & -7.65 & 18,16 & 15,99 & Granito & $-7,56$ & $-8,14$ & $-15,98$ & $-15,98$ \\
\hline Granito & 2.75 & $-3,30$ & $-9,49$ & $-9,05$ & Pizarra & 0,89 & $-7,96$ & $-4,46$ & $-7,48$ \\
\hline Pizarra & 2.96 & $-2,01$ & $-11,72$ & $-12,32$ & Pintura & $-11,19$ & $-5,51$ & 83,06 & 88,69 \\
\hline Terrazo & $17.50^{-}$ & $-14,59$ & $-19,29$ & $-18,78$ & Vidrio & $-8,00$ & $-7,45$ & 13,49 & 6,72 \\
\hline- & - & - & - & - & Papel & - & - & 14,85 & 17,82 \\
\hline
\end{tabular}

Fuente : Elaboración propia

El planteamiento de la Tabla 6 considera que los niveles de los atributos no están correlacionados entre sí, presentando, por tanto, ausencia de multicolinealidad para garantizar tanto la ortogonalidad entre dichos atributos, como la posibilidad de intercambio entre los distintos niveles. Por este motivo se seleccionaron finalmente la madera (parquet y tarima), los materiales cerámicos (azulejo, gres rústico, gres esmaltado y gres porcelánico), los pétreos naturales (pizarra, mármol y granito), la pintura, el terrazo, el vidrio y el papel, así como las mismas 2 aplicaciones de pavimento y de revestimiento en baño y cocina y, en salón y dormitorio. Por otro lado, se debe tener en cuenta que la distancia entre atributos mide la distancia en preferencia dentro de un mismo atributo, considerando siempre que las utilidades de diferentes atributos no son directamente comparables entre sí.

Tras analizar el comportamiento del consumidor, se concluye que existe una clara concordancia entre la aceptación del material para una aplicación concreta (baño y cocina o salón y dormitorio) y la utilidad maximizadora de la preferencia declarada de la percepción del encuestado/a. Es decir, que, si el material no es aceptado por el encuestado/a, éste no lo considerará apropiado para la aplicación propuesta. Los valores de utilidad medios muestran que la preferencia por un material de construcción para una aplicación concreta, marca en el encuestado/a un juicio de valor positivo sobre dicho material, repercutiendo en su utilidad global. 
La Tabla 7 muestra las estancias que marcan la decisión final del encuestado o de la encuestada y que desvelan su preferencia por cada uno de los materiales de construcción de la vivienda elegidos.

Tabla 7. Valores de utilidad importantes con la metodología del ACA

\begin{tabular}{|l|r|}
\hline Aplicación propuesta & Valores \\
\hline \hline Pavimento en baño & -11.00 \\
\hline Revestimiento en baño & -9.29 \\
\hline Pavimento en cocina & -10.64 \\
\hline Revestimiento en cocina & 7.22 \\
\hline Pavimento en salón & 29.34 \\
\hline Revestimiento en salón & 21.92 \\
\hline Pavimento en dormitorio & -7.65 \\
\hline Revestimiento en dormitorio & $-3,30$ \\
\hline
\end{tabular}

Fuente : Elaboración propia

En el caso de este estudio, los valores de utilidad claramente importantes para determinar la maximización de preferencia del uso residencial, se dan en primer lugar en pavimento para la estancia salón, cuando los materiales de construcción de la vivienda utilizados son parquet y tarima, y en revestimientos de esa misma estancia cuando el material utilizado es la pintura. En segundo lugar, en pavimentos en la estancia cocina, cuando los materiales de construcción de la vivienda utilizados son gres porcelánico o gres esmaltado y en revestimiento de esa misma estancia es el azulejo.

\section{Discusión y conclusiones}

La experiencia recabada en la investigación aquí reportada permite establecer la viabilidad de la validación de la construcción de un índice de calidad percibida subjetiva de los materiales de construcción de las viviendas de uso residencial en España, teniendo en cuenta su inexistencia respecto al análisis de la información sobre la estimación estandarizada de la utilidad media de los materiales escogidos por la demanda y los/as técnicos/as. De esta manera los/as expertos/as dispone de información valiosa ya que tanto la ubicación en el inmueble residencial como el origen de éstos, son condiciones determinantes para la valoración de dichos materiales, quedando penalizada su puntuación por la baja aceptación técnica percibida cuando, transcendiendo a un mayor grado de generalización, son usados en otras aplicaciones distintas a las consideradas como óptimas.

En la investigación aquí reportada, el análisis conjunto adaptativo permite realizar una entrevista cuantitativa de forma individual y adaptable en tiempo real en base a las respuestas de los/las participantes, siendo más corta y fácil de contestar gracias a que ofrece todas las posibles combinaciones de los atributos, evitando al participante una sobrecarga de información al presentárselos de dos en dos en vez de todos a la vez. De esta forma, la variable más importante a controlar son las excesivas contestaciones facilitadas al participante cuando se da un gran número de factores en la investigación a tratar.

Concretamente, la importancia de la percepción del material asociado a la estancia de la vivienda donde se ubique el material debe ser el centro de atención, dado que la demanda está dispuesta a pagar más por una vivienda construida con materiales versátiles (como por ejemplo gres porcelánico, gres rústico o gres esmaltado, mármol y parquet o tarima), que por aquella construida con materiales inaceptables (como barro cocido o terrazo). De hecho, se deben considerar dichos atributos como una herramienta estratégica para mejorar el valor-utilidad de percepción de la vivienda. De manera que en el caso de las preferencias y opiniones de los ciudadanos y los técnicos expertos en el sector de la construcción en la estimación de las tasas marginales de sustitución de la utilidad de los materiales de construcción de los inmuebles residenciales, la técnica del análisis conjunto adaptativo 
en este contexto se presenta como una herramienta metodológica que posibilita conocer la configuración del bien inmueble que resulta más atractivo para cualquier consumidor, permitiendo realizar estudios de segmentación de mercado reduciendo la complejidad del cuestionario. Por este motivo, constructores, promotores, empresas de construcción, arquitectos/as y los arquitectos técnicos/as se deben orientar cada vez más hacia cualquier consumidor potencial en la elección de los materiales de construcción de una vivienda, y así poder maximizar y optimizar la percepción de su utilidad, lo que les permite ser cada vez más competitivos/as y tener ventajas para crecer y desarrollarse.

Respecto a la versatilidad de los materiales, observamos que cada participante reacciona de forma homogénea, con una percepción claramente negativa, es decir, si no son considerados apropiados y socialmente admitidos para una localización y ubicación concreta, circunstancia relacionada con factores de tipo económico, de mantenimiento y de durabilidad. Considerada la importancia que la demanda da a una ubicación específica, su percepción de la calidad técnica y de la calidad estética de un material son resultado de dos variables sinérgicas. De hecho, la forma en la que se percibe la calidad de los materiales según su posición en la vivienda, ha puesto en relieve, que esta apreciación no se corresponde con una valoración objetiva basada en criterios técnicos compartidos, confirmando que la demanda está influenciada por una serie de factores en el momento de evaluar la calidad interior de una vivienda.

Las puntuaciones máximas otorgadas al pavimento, del salón y dormitorios, se dan en la madera (parquet y tarima), mientras que, en el baño y la cocina, la puntuación más elevada la adquieren los materiales cerámicos (gres porcelánico, gres rústico y gres esmaltado). La elección de los materiales mencionados, bastante predecible, confirma el uso habitual de un determinado tipo de material, puntuado con un valor más alto, independientemente de sus especificaciones técnicas. No obstante, es importante destacar que la falta de conocimiento real de las características técnicas de estos materiales, propician unos resultados indicativos de la confusión existente entre la estética y la calidad percibidas. Asimismo, resulta más fácil para los usuarios reconocer pétreos naturales que distinguir entre diferentes tipos de materiales cerámicos. Como excepción, con una puntuación mínima, se permite utilizar la pizarra para la pavimentación del baño.

Acerca de las puntuaciones máximas de los revestimientos, se observa que en el baño y la cocina sólo se admiten materiales versátiles, siendo por ejemplo los cerámicos en la estancia cocina (gres rústico) o el pétreo natural (mármol) en la estancia baño. Para el salón y los dormitorios, la pintura predomina en casi la totalidad de las ocasiones por ser una opción más barata y presentar diversidad de terminaciones, admitiéndose como excepciones el vidrio y el papel. Desde el punto de vista operativo, estas elecciones tienen un particular impacto en el cálculo de la estimación del valor de mercado, ya que los resultados ponen de manifiesto que, desde la percepción subjetiva, los materiales de construcción de la vivienda se agrupan por grado de versatilidad. En consecuencia, el concepto "valor-utilidad" de un material de construcción adecuado para un revestimiento, asume un sentido más preciso, (aunque no del todo racional) a la luz de la teoría del comportamiento del consumidor.

Por otro lado, respecto a la maximización de la utilidad de los pavimentos la estancia salón, la adquiere la madera, seguido del mármol, mientras que en la maximización de la utilidad de las mismas estancias en revestimientos la adquiere la pintura para el salón, y admitiendo como materiales alternativos el papel y el vidrio. En cuanto a la cocina, los revestimientos están marcados por los materiales cerámicos, que adquieren un mayor nivel de utilidad, aunque en menor medida en comparación con los casos anteriores.

ACE, 15 (43) CC BY-ND 3.0 ES | UPC Barcelona, España | Preferencias de la demanda sobre los materiales de construcción del inmueble residencial: caso de estudio con el Análisis Conjunto Adaptativo. DOI: http://dx.doi.org/10.5821/ace.15.43.9245 
En definitiva, la posibilidad de conocer la opinión de la demanda o técnico/a en la materia sobre la percepción de la calidad subjetiva de los materiales de construcción de la vivienda, no corresponde exclusivamente al análisis conjunto adaptativo ni a otros métodos des-composicionales. Sin embargo, esta investigación es pionera en dar un primer paso hacia el conocimiento del valor- utilidad en este tipo de percepción. En ella, se disminuyen al mínimo algunos de los efectos asociados a la subjetividad de las opiniones verificadas, utilizando otros métodos menos formalizados como la encuesta de opinión. Asimismo, esta técnica ha permitido una adaptación al caso de estudio, demostrando así ser lo suficientemente flexible, con enorme potencialidad y que permite seguir un modelo de desarrollo sustentable.

\section{Agradecimientos}

Los autores agradecen a los revisores externos sus aportaciones que han permitido mejorar este artículo. También, al Dottorato di Ricerca in Ambiente e Territorio Indirizzo Estimo e Valutazioni Economiche por brindar asistencia en esta investigación, especialmente a la Dra. Marina Bravi y al Dr. Riccardo Roscelli.

\section{Autoría}

Ambos autores han desarrollado la conceptualización de la problemática. La primera autora (como doctoranda) ha aprendido la metodología y el manejo práctico de la técnica del ACA, ha interpretado el análisis de los resultados, ha escrito el artículo y ha realizado la revisión. El segundo auto (como director de la tesis doctoral) ha establecido la metodología a seguir, revisando la interpretación, el análisis de los resultados, el escrito y la edición del artículo.

Conflicto de intereses: Los autores declaran que no hay conflicto de intereses.

\section{Bibliografía}

Agarwal, M. y Green, P. (1991). Adaptive Conjoint Analysis Versus Self-Explicated Models: Some Empirical Results. International Journal of Research in Marketing, 8(2), 141-146. DOI: https://doi.org/10.1016/0167-8116(91)90021-X

Alaraji, K.A. y Jusan, M.B. (2014). Assessment of perceived flexibility in house design using conjoint analysis (CA). International Journal of Applied Engineering Research, 9(14), 2473-2486. Recuperado de https://www.ripublication.com/Volume/ijaerv9n14.htm

Arboretti, R. (2003). Adaptive and Full profile conjoint analysis: alcune considerazioni e software statistici a confront. [Biblioteca di scienze statistiche]. Recuperado de http://paduaresearch.cab.unipd.it/7295/1/WP 2003 4.pdf

Herrmann, A.; Schmidt, D. y Huber, F. (2000). Adaptive Conjoint Analysis: Understanding the Methodology and Assessing Reliability and Validity. In: Conjoint Measurement. Springer, Berlin, Heidelberg. DOI: https://doi.org/10.1007/978-3-662-06395-8 11

Arning, K. y Ziefle, M. (2015). Get that Camera Out of My House! Conjoint Measurement of Preferences for Video-Based Healthcare Monitoring Systems in Private and Public Places. In: Geissbühler, A. Demongeot, J. Mokhtari, M. Abdulrazak, B. Aloulou, H. (eds) Inclusive Smart Cities and e-Health. ICOST 2015. Lecture Notes in Computer Science, Springer, Cham, 9102, 152-164. DOI: https://doi.org/10.1007/978-3-319-19312-0 13

ACE, 15 (43) CC BY-ND 3.0 ES | UPC Barcelona, España | Preferencias de la demanda sobre los materiales de construcción 
Bae, H. (2011). Urban stream restoration in Korea: Design considerations and residents. Urban Forestry \& Urban Greening, 10(2), 119-126. DOI: https://doi.org/10.1016/j.ufug.2011.02.001

Banfi, S.; Farsi, M.; Filippini, M. y Jakob, M. (2008). Willingness to pay for energy-saving measures in residential buildings. Energy Economics, 30(2), 503-516. DOI: https://doi.org/10.1016/j.eneco.2006.06.001

Ferreira, S. (2011). Análisis Conjunto. Teoría, campos de aplicación y conceptos inherentes. Estudios y perspectivas en turismo, 20(2), 341-366. Recuperado de https://www.estudiosenturismo.com.ar/

Franke, M. y Nadler, C. (2019). Energy efficiency in the German residential housing market: Its influence on tenants and owners. Energy Policy, 128, 879-890. DOI: https://doi.org/10.1016/j.enpol.2019.01.052

Goodin, Robert. E. (1995). La utilidad y el bien. Compendio de Ética. Recuperado de file:///C:/Users/Silvia/Downloads/Compendio de tica Peter Singer.pdf

Goossen, M. y Langers, F (2000). Assessing quality of rural areas in the Netherlands: finding the most important indicators for recreation. Landscape and Urban Planning, 46(4), 241-251. DOI: https://doi.org/10.1016/S0169-2046(99)00058-4

Green, P. y Srinivasan, V. (1990). Conjoint Analysis in Marketing: New Developments with Implications for Research and Practice. Journal of marketing, 54 (4), 3-19. DOI: https://doi.org/10.2307/1251756

Grönroos, C. (1984). A service quality model and its marketing implications. European Journal of Marketing, 18(4), 36-44. DOI: https://doi.org/10.1108/EUM0000000004784

Gustafsson, A.; Herrmann, A. y Huber. F. (2007). Conjoint measurement: Methods and applications. DOI: $\underline{\text { https://doi.org/10.1007/978-3-540-71404-0 }}$

Hagishima, A.; Park, M. y Tanimoto, J. (2012). Questionnaire survey on preference of environmental factors of residential buildings. Journal of Environmental Engineering, 77(676), 523-531. DOI: https://doi.org/10.3130/aije.77.523

Heinzle, S.L.; Boey, A. y Low, M. (2013). The Influence of Green Building Certification Schemes on Real Estate Investor Behaviour: Evidence from Singapore. Urban Studies, 50(10), 1970-1987. DOI: https://doi.org/10.1177/0042098013477693

Hensher, D.; Rose, J. y Greene, W. (2005). Applied Choice Analysis, DOI: https://doi.org/10.1007/9781316136232

Hildebrandt, J.; Brauner, P. y Ziefle, M. (2015). Smart Textiles as Intuitive and Ubiquitous User Interfaces for Smart Homes. Lecture Notes in Computer Science, 9194, 423-434. DOI: https://doi.org/10.1007/978-3-319-20913-5 39

Hogarth, Robin. M. (1987). Judgment and Choice: The psychology of decision (2a ed.). University of Minnesota: Wiley.

Huertas, R.; Gálvez, J.C.; Martínez, F. J. y Esteban, I. (2014). Propuesta metodológica mediante diseños Box-Behnken para mejorar el rendimiento del análisis conjunto en estudios experimentales de mercado. Revista Española de Investigación de Marketing ESIC, 18(1), 57-66. DOI: https://doi.org/10.1016/S1138-1442(14)60006-1 
Fitri, M. et al. (2017). A topology of residents' based on preferences for sustainable riparian settlement in Palembang, Indonesia. En Proceedings of MATEC Web of Conferences, Santika, Indonesia. DOI: https://doi.org/10.1051/matecconf/201710105025

Jay, I y Bowen, P (2011). What residents value in low-cost housing schemes: some South African concepts. Building research and information. 39(6), 574-588. DOI: https://doi.org/10.1080/09613218.2011.617082

Johnson, E. J. y Meyer, R. J. (1984). Compensatory choice models of non-compensatory processes: The effect of Varying Context. Journal of Consumer Research, 11(1), 528-541. https://doi.org/10.1086/208989

Johnson, R. M. (1987). Accuracy of Utility Estimation in ACA. Sequim, WA: Sawtooth Software. Sawtooh Software, Inc. Recuperado de https://www.sawtoothsoftware.com/download/techpap/acaaccur.pdf

Johnson, R. M. (1989). Assessing the Validity of Conjoint Analysis. En Proceedings of the Sawtooth Conference on Perceptual Mapping, Conjoint Analysis, and Computer Interviewing. Ketchum, ID: Sawtooth Software, Inc.

Johnson, R. M. (1991). Comment on 'Adaptive Conjoint Analysis: same caveats and suggestions. Journal of marketing research. 28, 223-225. DOI: https://doi.org/10.1177/002224379102800209

Lambin, J. J. y Peeters, R. (1981). La gestión del marketing de las empresas. Madrid: ICE.

Lancaster, K. (1990). The economics of product variety: A survey. Marketing Science, 9(3), 189-206. DOI: https://doi.org/10.1287/mksc.9.3.189

Lee, B. K.; Sohn, S. Y. y Yang, S. (2014). Design guidelines for the Dashilar, Beijing open green space redevelopment project. Urban Forestry \& Urban Greening, 13(2), 385-396. DOI: https://doi.org/10.1016/j.ufug.2013.12.008

Macias, N. y Knowles, C. (2011). Examining the effect of environmental certification, wood source, and price on architects' preferences of hardwood flooring. Silva Fennica, 45(1), 97-109. DOI: https://doi.org/10.14214/sf.34

Maeng, K. B., Jung, J. y Koo, Y. (2019). Quantitative analysis of consumer preferences of windows set in South Korea: The role of energy efficiency levels. Energies, 12(9), 1816. DOI: https://doi.org/10.3390/en12091816

Mansour, O. E. y Radford, S. K. (2016). Rethinking the environmental and experiential categories of sustainable building design, a conjoint analysis. Building and Environment, 98, 47-54. DOI: 10.1016/j.buildenv.2015.12.014

Marmolejo, C. y Ruiz, M. (2013). Using Choice-based-experiments to support real estate design decisions. Journal of European Real Estate Research, 6(1), 63-89. DOI: https://doi.org/10.1108/17539261311312979

Marmolejo, C. y Ruiz, M. (2014). El análisis conjunto como herramienta de soporte en la toma de decisiones urbanísticas: dos casos de estudio en Cataluña. Ciudad y territorio. Estudios territoriales. XLVI (182), 1-19. Recuperado en: https://dialnet.unirioja.es/servlet/articulo?codigo=4899827 
Marmolejo, C.; Olivares, A.; Montero, J. y De Paolini, M. (2014). El uso del análisis conjunto en el diseño de nuevos sistemas de transporte: el caso del auto tren del Distrito Cultural Universitario en Guadalajara. Revista de la Facultad de Arquitectura Universidad Autónoma de Nuevo León. 8, 29-50 Recuperado en: https://dialnet.unirioja.es/servlet/articulo?codigo=4737109

Marmolejo, C. y Bravi, M. (2017). Does the Energy Label (EL) Matter in the Residential Market? A Stated Preference Analysis in Barcelona. Buildings, 7(53), 2-16. DOI: https://doi.org/10.3390/buildings7020053

Marmolejo, C.; García, A. y Spairani, S. (2020). Panorama de la certificación energética en España. La perspectiva de los principales agentes del engranaje inmobiliario residencial. Ciudad y territorio. Estudios territoriales. LIII (205), in press.

Morrow, H.A.; Irwin, E.G. y Roe, B. (2004). Consumer preference for neotraditional neighborhood characteristics. Housing Policy Debate, 15(1), 171-202. DOI: https://doi.org/10.1080/10511482.2004.9521498

Nordh, H. (2012). Quantitative methods of measuring restorative components in urban public parks. Journal of Landscape Architecture, 7(1), 46-53. DOI: https://doi.org/10.1080/18626033.2012.6937800

Oliphant, k., Eagle, T.C.; Louviere, J.J. y Anderson, D.A. (1992). Cross-task comparison of ratings-based and choice-based conjoint. En Proceedings of Sawtooth Software Conference, Idaho, 383-404. Recuperado de https://www.sawtoothsoftware.com/support/technical-papers/conferenceproceedings/proceedings1992

Robinson, P.J. (1980). Application of conjoint analysis to pricing problems. En D.B. Montgomery and D.R. Wittink (Eds.), Proceedings of the first ORSA / TIMS special interest conference on market measurement and analysis (pp193-205). Cambridge, MA: Marketing Science Institute.

Rotaris, L. (2002). La stima della domanda di trasporto merci attraverso la tecnica delle Stated Preferences: apetti teorico. En D. Romeo, Domanda di transporto merci e preferenze dichiarate Freight transport demand and Stated Preferences Experiments. Milano, Italia: Franco Angeli.

Sawtooth Software Inc. (1985-2010), applicative SMRTV.4.20.2. Sequim: Washington. Recuperado en: www.sawtoothsoftware.com/download/techpap/aca-tech.pdf

Rofe, Y.; Pashtan, T. y Hornik, J (2017). Is there a market for sustainable urbanism? A conjoint analysis of potential homebuyers in Israel. Sustainable cities and society, 30, 162-170. DOI: https://doi.org/10.1016/j.scs.2017.01.011

Simonotti, M. (2006). Metodi di stima immobiliare. Applicazione degli standorà internazionali, Palermo: Flaccovio. Recuperado en: https://www.darioflaccovio.it/abstracts/9788877586865/metodi-di-stimaimmobiliare-simonotti 9788877586865.pdf

Som, A. y Lee, Y.H. (2012). The joint effects of choice assortment and regulatory focus on choice behaviour. Annual Review of Psychology, 41(1), 1-20. DOI: https://doi.org/10.1016/j.jjresmar.2012.01.003

Spairani, S. y Roca, J. (2013). Use of the Conjoint Analysis technique to establish the perceived quality of building materials in the residential use. Preliminary studies. En Proceedings of the 20th Annual European Real Estate Society Conference in Vienna (no 98), Austria, July 2013. Recuperado de https://ideas.repec.org/p/arz/wpaper/eres2013 67.html

ACE, 15 (43) CC BY-ND 3.0 ES | UPC Barcelona, España | Preferencias de la demanda sobre los materiales de construcción 
Spairani Berrio, S. (2016). Valor de mercado y percepción de la calidad de los materiales de construcción: aplicación en pavimentos y revestimientos interiores en el uso residencial (Tesis doctoral), Universidad Politécnica de Cataluña. Barcelona. Recuperado de http://hdl.handle.net/2117/98122

Spairani, S. et al. (2019). The incidence of energy efficiency certificates in the residential market: the perspective of real estate agents. En Proceedings of the XV Inter. Cong. on Environmental, Cultural, Economic and Social Sustainability, Vancouver, Canada. Recuperado de http://vertice.cpd.ua.es/200824, http://hdl.handle.net/10045/85230

Thaler, R.H. (1985). Mental Accounting and Consumer Choice. Marketing Science, 4(3), 199-214. DOI: https://doi.org/10.1287/mksc.4.3.199

Timmermans, H. y Katoshevski, R. (2001). Using Conjoint Analysis to Formulate User-centred Guidelines for Urban Design: The Example of New Residential Development in Israel. Journal of urban design. 6(1), 37-53. DOI: https://doi.org/10.1080/13574800120032888

Kahneman, D. y Tversky, A. (1979). Prospect Theory: An Analysis of Decision under Risk. Econometrica, 47(2), 263-291. DOI: https://doi.org/10.2307/1914185

Zeithaml, V. (1988). Consumer perceptions of price, quality, and value: a means end model and synthesis of evidence. Jornal of Marketing, 52(3), 2-22. DOI: https://doi.org/10.1177/002224298805200302

Zhang, L.-X., Chen, T., Wang, Y.-M. y Zhu, J. (2012). Research on housing product positioning based on conjoint analysis. Dongbei Daxue Xuebao/Journal of Northeastern University, 33(5), 752-756. Recuperado

de https://www.researchgate.net/publication/297466009 Research on housing product positioning b ased on conjoint analysis

ACE, 15 (43) CC BY-ND 3.0 ES | UPC Barcelona, España | Preferencias de la demanda sobre los materiales de construcción 